\title{
Sex influences eQTL effects of SLE and Sjögren's syndrome-associated genetic polymorphisms
}

\author{
Magdalena Lindén ${ }^{1}$, Jorge I. Ramírez Sepúlveda', Tojo James², Gudny Ella Thorlacius', Susanna Brauner², \\ David Gómez-Cabrero $3,4,5,6$, Tomas Olsson², Ingrid Kockum² and Marie Wahren-Herlenius ${ }^{1 *}$ (D)
}

\begin{abstract}
Background: Systemic lupus erythematosus (SLE) and primary Sjögren's syndrome (pSS) are autoimmune disorders characterized by autoantibodies, dysregulated B cells, and notably high female-to-male incidence ratios. Genomewide association studies have identified several susceptibility SNPs for both diseases. Many SNPs in the genome are expression quantitative trait loci (eQTLs), with context-dependent effects. Assuming that sex is a biological context, we investigated whether SLE/pSS SNPs act as eQTLS in B cells and used a disease-targeted approach to understand if they display sex-specific effects.
\end{abstract}

Methods: We used genome-wide genotype and gene expression data from primary B cells from 125 males and 162 females. The MatrixEQTL R package was used to identify eQTLs within a genomic window of $2 \mathrm{Mb}$ centered on each of 22 established SLE and/or pSS susceptibility SNPs. To find sex-specific eQTLs, we used a linear model with a SNP * sex interaction term.

Results: We found ten SNPs affecting the expression of 16 different genes (FDR < 0.05). rs7574865-INPP1, rs7574865-MYO1B, rs4938573-CD3D, rs11755393-SNRPC, and rs4963128-PHRF1 were novel observations for the immune compartment and B cells. By analyzing the SNP * sex interaction terms, we identified six genes with differentially regulated expression in females compared to males, depending on the genotype of SLE/pSS-associated SNPs: SLC39A8 (BANK1 locus), CD74 (TNIP1 locus), PXK, CTSB (BLK/FAM167A locus), ARCN1 (CXCR5 locus), and DHX9 (NCF2 locus).

Conclusions: We identified several unknown sex-specific eQTL effects of SLE/pSS-associated genetic polymorphisms and provide novel insight into how gene-sex interactions may contribute to the sex bias in systemic autoimmune diseases.

Keywords: Sjögren's syndrome, SLE, Autoimmunity, Sex difference, eQTL

\section{Background}

Autoimmune disorders such as SLE and pSS have a multifactorial etiology. Multiple environmental as well as intrinsic risk factors interplay with each other and an individual's genetic makeup in different combinations over time in order to cause disease. Smoking, infection, and textile dust have been identified as extrinsic factors interacting with the host genome to increase the risk of

\footnotetext{
* Correspondence: Marie.Wahren@ki.se

${ }^{1}$ Unit of Experimental Rheumatology, Department of Medicine, Karolinska

University Hospital, Karolinska Institutet, Stockholm, Sweden

Full list of author information is available at the end of the article
}

autoimmune disease [1-3], while adolescent obesity is the only intrinsic factor to date with a proven genetic interaction to influence autoimmune disease risk [4].

The majority of autoimmune diseases, and especially the rheumatic diseases, are more common in women than in men [5]. Systemic lupus erythematosus (SLE) and primary Sjögren's syndrome (pSS) are clinically and molecularly related systemic autoimmune disorders characterized by autoantibodies and dysregulated B cells. Both diseases have among the highest observed femaleto-male ratios compared to other autoimmune conditions (approximately 9:1 for SLE [6] and 9:1 ranging to $20: 1$ for pSS $[7,8])$. The roles of sex hormones and the 
sex chromosomes have been extensively investigated [9-11], but the strong sex bias remains poorly understood.

Although the etiology of SLE and pSS is unknown, genetics play a significant role. Through genome-wide association studies (GWAS), a growing number of single nucleotide polymorphisms (SNPs) that confer disease susceptibility are being identified for both diseases [12-18]. There is a certain overlap of genetic risk variants between different autoimmune disorders, also between rheumatic diseases, and many of the loci implicated in SLE have also been identified in pSS $[17,18]$. Most of the diseaseassociated SNPs of complex conditions are located in non-coding sequences and are thought to affect regulatory genetic elements, leading to altered transcriptional activity, splicing, and epigenetic marks [19, 20]. Expression quantitative trait loci (eQTLs) are widespread in the genome [21] and likely to account for a substantial part of the causal genetic effects contributing to disease [22, 23]. In fact, several publications show that the expression of a number of genes is affected by SNPs in SLE- and pSSassociated regions. It is becoming increasingly clear that a large proportion of eQTLs present context-dependent effects relating to biological factors such as cell type and activation state [24-26].

Investigating the functional outcome of genetic associations linked to disease will give novel insight to pathogenesis. As there is no difference of the frequency of these pSS and SLE disease-associated SNPs between women and men in the general population, but a much higher likelihood for the diseases to develop in women carrying the SNPs, we hypothesized that the context female sex would influence the functional impact of the genetic polymorphism. Previous investigations of sexually dimorphic eQTLs have been performed in heterogeneous, whole tissue samples $[27,28]$, and given the central role of B cells in both pSS and SLE pathogenesis, we performed our analysis in an expression dataset derived from purified B cells. We first investigated whether SLE/pSS disease-associated SNPs act as eQTLs in B cells. Further, to better understand the difference between sexes in systemic autoimmune diseases and assuming that sex is a biological context, we thereafter applied a statistical model that takes the interaction between SNPs and sex into account and examined whether eQTL effects differ between women and men in the disease-associated loci.

\section{Methods}

Study subjects, gene expression array data, and SNP genotypes

Microarray-based $\mathrm{CD} 19^{+}$B cell mRNA expression and genotype data from 287 healthy volunteers (age range 18-62 years; median age 33.1 years) was obtained from a study published by Fairfax et al. [24]. Sampling and laboratory procedures are described in detail elsewhere [24]. In short, the Illumina HumanHT-12 v4 BeadChip gene expression array platform was used for total RNA quantification and the expression data was obtained as $\log -2$ transformed values. The Illumina Human OmniExpress12v1.0 BeadChips, NCBI36 Build, were used for genomic DNA genotyping, and upon granted access, genotype data was downloaded from the European Genome-Phenome Archive as AGCT-coded SNP data.

\section{SLE- and pSS-associated SNPs}

The SNPs were chosen from the current literature of genetic association studies in SLE and/or pSS, with the criteria of reaching genome-wide significance $\left(p<5.0 \times 10^{-8}\right)$ and being the first reported and/or top-associated SNP within the associated region, and/or being genotyped in the Fairfax et al.'s study [24]. SNPs in the HLA region were excluded due to low allele frequencies in the dataset $(\geq 3$ individuals for each associated $H L A$ allele were required per genotype group). For SNPs that were not present on the Illumina genotyping chip used by Fairfax et al., proxy SNPs with high linkage disequilibrium (LD) $\left(r^{2}>0.8\right)$ were identified using the SNAP Proxy search tool (SNP dataset: 1000 Genomes Pilot 1; Population panel: Caucasian (CEU); $r^{2}$ threshold 0.8; distance limit 500) [29]. Proxy SNPs and $r^{2}$ values in relation to the corresponding SLE/ pSS-associated SNP are found in Table 1. $r^{2}$ values for SLE- and pSS-associated SNPs in the same region were retrieved using HaploReg v.4.1 (1000 Genomes Phase 1, CEU population) [30].

\section{Imputation of sex of the genotyped individuals and confirmation by expression analysis}

We defined the sex of each individual using the "- -impute-sex" command in PLINK v. 1.07. This tool imputes the sex codes based on SNP data by estimating the heterozygosity rates of the $\mathrm{X}$ chromosome [31]. To further confirm the sex of the samples, we utilized the MicroArray Sample Sex Identifier R package (massiR) which predicts the sex of a given sample based on the variance of expression levels of $\mathrm{Y}$ chromosome genes probes. The probes with higher expression variance across the samples will be indicative of sex variation and, thus, can be used for sex classification.

When we defined the sex of the samples with PLINK or massiR, the same results with a perfect match was obtained.

Population substructure and principal component analysis Genotypes for 3736 ancestry informative SNPs were used for principal component analysis (PCA) by running the smartpca program from Eigensoft using standard settings, Additional file 1: Figure S1. Five population 
Table 1 List of pSS- and/or SLE-associated SNPs used in the eQTL analyses

\begin{tabular}{|c|c|c|c|c|c|c|c|c|c|}
\hline Chr & SNP & $\begin{array}{l}\text { Proxy SNP for top } \\
\text { associated SNP }\left(r^{2}\right)\end{array}$ & Gene region & $\begin{array}{l}\text { MAF }^{\mathrm{b}} \\
\text { males/females }\end{array}$ & $\begin{array}{l}\text { Disease } \\
\text { association }\end{array}$ & $\begin{array}{l}\text { Disease } \\
\text { risk allele }^{a}\end{array}$ & OR & Assoc P & Reference \\
\hline 1 & rs3024505 & & $1 L-10$ & $0.17 / 0.14$ & SLE & $A$ & 1.19 & $4.00 \times 10^{-8}$ & Gateva et al. [14] \\
\hline 1 & rs10911363 & rs12753665 (0.821) & NCF2 & $0.26 / 0.29$ & SLE & $\mathrm{T}(\mathrm{A})$ & 1.23 & $2.87 \times 10^{-10}$ & Cunninghame Graham et al. [16] \\
\hline 2 & rs1990760 & & $|F| H \mid$ & $0.42 / 0.42$ & SLE & $\mathrm{T}$ & 1.17 & $1.63 \times 10^{-8}$ & Cunninghame Graham et al. [16] \\
\hline 2 & rs7574865 & & STAT4 & $0.27 / 0.20$ & SLE & $\mathrm{T}$ & 1.57 & $1.40 \times 10^{-10}$ & Gateva et al. [14] \\
\hline 3 & rs485497 & & IL12A & $0.49 / 0.51$ & pSS & A & 1.3 & $1.17 \times 10^{-10}$ & Lessard et al. [17] \\
\hline 3 & rs6445975 & & PXK & $0.23 / 0.26$ & SLE & $C$ & 1.25 & $7.10 \times 10^{-9}$ & Harley et al. [13] \\
\hline 4 & rs10516487 & rs4637409 (1) & BANK1 & $0.35 / 0.30$ & SLE & $G(A)$ & 1.38 & $3.74 \times 10^{-10}$ & Kozyrev et al. [15] \\
\hline 5 & rs7708392 & rs10036748 (1) & TNIP1 & $0.26 / 0.25$ & SLE & $C(T)$ & 1.27 & $3.80 \times 10^{-13}$ & Gateva et al. [14] \\
\hline 6 & rs6568431 & & PRDM1/ATG5 & $0.38 / 0.40$ & SLE & A & 1.2 & $7.10 \times 10^{-10}$ & Gateva et al. [14] \\
\hline 6 & rs5029939 & & TNFAIP3 & $0.04 / 0.05$ & SLE & G & 2.29 & $2.86 \times 10^{-12}$ & Graham et al. [58] \\
\hline 6 & rs11755393 & & UHRFIBP1 & $0.32 / 0.34$ & SLE & G & 1.17 & $2.20 \times 10^{-8}$ & Gateva et al. [14] \\
\hline 7 & rs2070197 & rs10488631 (0.938) & IRF5/TNPO3 & $0.10 / 0.10$ & SLE & $C(C)$ & 1.88 & $5.80 \times 10^{-24}$ & Gateva et al. [14] \\
\hline 7 & rs4728142 & & IRF5/TNPO3 & $0.45 / 0.46$ & pSS & $C(A)$ & 1.31 & $2.73 \times 10^{-19}$ & Lessard et al. [17] \\
\hline 7 & rs849142 & & JAZF1 & $0.49 / 0.50$ & SLE & $\mathrm{T}$ & 1.19 & $1.50 \times 10^{-9}$ & Gateva et al. [14] \\
\hline 8 & rs13277113 & & BLK/C8orf13 & $0.25 / 0.25$ & SLE & A & 1.39 & $1.00 \times 10^{-10}$ & Hom et al. [12] \\
\hline 8 & rs 2736345 & rs922483 (1) & BLK/C8orf13 & $0.29 / 0.29$ & pSS & $\mathrm{G}(\mathrm{T})$ & 1.16 & $4.97 \times 10^{-10}$ & Lessard et al. [17] \\
\hline 8 & rs7829816 & & LYN & $0.19 / 0.21$ & SLE & $\mathrm{T}$ & 1.23 & $5.40 \times 10^{-9}$ & Harley et al. [13] \\
\hline 11 & rs2732552 & & CD44, PDHX & $0.40 / 0.49$ & SLE & $C$ & 1.17 & $1.82 \times 10^{-9}$ & Lessard et al. [59] \\
\hline 11 & rs7119038 & rs4938573 (0.865) & CXCR5 & $0.22 / 0.22$ & pSS & $A(T)$ & 1.21 & $1.10 \times 10^{-8}$ & Lessard et al. [17] \\
\hline 11 & rs4963128 & & IRF7/PHRF1/KIAA1542 & $0.36 / 0.29$ & SLE & C & 1.22 & $3.00 \times 10^{-10}$ & Harley et al. [13] \\
\hline 16 & rs11644034 & & IRF8 & $0.22 / 0.23$ & SLE & G & 1.22 & $2.72 \times 10^{-9}$ & Lessard et al. [60] \\
\hline 16 & rs9888739 & & ITGAM-ITGAX & $0.09 / 0.12$ & SLE & $\mathrm{T}$ & 1.62 & $1.61 \times 10^{-23}$ & Harley et al. [13] \\
\hline
\end{tabular}

Chr chromosome, SNP single nucleotide polymorphism, OR odds ratio, Assoc $P p$ value for the disease association

${ }^{a}$ Allele in parenthesis denotes the corresponding allele for the proxy SNP

${ }^{\mathrm{b}}$ Minor allele frequency (MAF) in this study dataset

outliers were identified. Due to this low number, none of them was removed from the analysis. None of the PCs was significant. There was no need to correct the analysis for PCA vectors, since the population was so homogenous.

\section{Filtering of expression data}

Genomic positions of the Illumina probes were retrieved from NCBI Reference Sequence build 36 utilizing the Illumina HumanHT-12 v4 BeadChip probe information file obtained from Illumina. We obtained SNP coordinates in NCBI build 36 by using the UCSC liftOver tool, http://genome.ucsc.edu/cgi-bin/hgLiftOver (from NCBI build 38 coordinates given in dbSNP) and applying the default parameters. Genes within the range of $\pm 1 \mathrm{Mb}$ of the SNP of interest were identified using BioMart (http://www.biomart.org/), and probe IDs of the corresponding genes were retrieved from the expression datasets. Among the $\mathrm{Y}$ chromosome non-recombining region genes with the highest male-to-female expression ratio in the $\mathrm{B}$ cell expression dataset, EIF1AY had the highest mean log-2 value in females. We therefore used it to set the threshold for gene expression in the whole dataset. Probes with $\geq 20 \%$ of samples below the expression cutoff were excluded from further analysis. We normalized the $\log 2$ expression values by transformation to $z$ scores.

\section{General cis eQTL analysis}

First, using $\mathrm{R}$, we investigated the regression assumptions of linearity, homoscedasticity, and normality of the transformed and scaled expression data for all genes $1 \mathrm{Mb}$ up- and downstream of each included SNP, prior to applying the linear model in subsequent eQTL analyses. This genomic distance is commonly used for studies of cis-eQTL effects.

For analysis of eQTL effects in the full cohort, we used the standard method applied in the MatrixEQTL R package [32]. We used sex as a covariate and did not take trans eQTL associations into account. For significance, we used a false discovery rate (FDR) of $<0.05$ as a cutoff. The number of probes and genes analyzed for each SNP are given in Additional file 2: Table S1. 
Analysis of differential cis eQTL effects in males and females An interaction term to estimate the joint effect of SNP genotype and sex was added to the regression model and was implemented using the "lm" function in $\mathrm{R}$ as follows:

$\operatorname{lm}($ Gene Expression $\sim$ SNP genotype + Sex + SNP genotype* Sex).

The "SNP genotype" factor in the argument of the $\mathrm{lm}$ model formula was coded based on the additive genetic effect with values $0,1,2$, for the number of risk alleles, and "Sex" with values 0,1 , for male or female. Normalized $z$ score values were used for "Gene Expression."

In order to exclude potential outlier-driven results and to correct for multiple comparisons, we permuted each SNP genotype 10,000 times and calculated a permutationbased FDR for the SNP genotype ${ }^{*}$ Sex interaction term coefficient according to the SAMseq method [33], which in turn is based on the FDR calculation described by Storey and Tibshirani [34]. The permuted $p$ value and FDR cutoff was set to $<0.05$.

\section{Comparisons with published eQTLs}

HaploReg v.4.1 (1000 Genomes Phase 1, CEU population) [30] was searched for published eQTL results in any tissue to compare with eQTLs found in this study. Any SNP with $r^{2}>0.8$ and displaying an eQTL effect was considered as a published result.

\section{LD SNP interaction analysis}

SNPs in LD with the sex-biased eQTLs were identified using the SNAP Proxy search tool (SNP dataset: 1000 Genomes Pilot 1; Population panel: Caucasian (CEU); $r^{2}$ threshold 0.8; distance limit 500) [29] and extracted from the Fairfax et al. genotype data. LD SNPs and $r^{2}$ values in relation to the corresponding SLE-associated SNP are found in Additional file 3: Table S2. For the rs12753665-DHX9 association only, a SNP with $r^{2}=0.6$ was available. rs13277113 and rs922483 are in themselves in high LD with each other, and only one additional proxy SNP could be found for both of them. Therefore, six proxy SNPs were included in the analysis.

\section{Type 1 diabetes-related polymorphisms}

Analysis of differential cis eQTL effects in males and females of polymorphisms associated with type 1 diabetes (T1D) was performed as described for SLE/pSS SNPs described above. SNPs were chosen from the current literature of genetic association studies in T1D [35], with the criteria of reaching genome-wide significance $\left(p<5.0 \times 10^{-8}\right)$ and being the first reported and/or topassociated SNP within the region. We also requested the SNP was not associated with any other disease with higher female:male ratio, leaving 15 SNPs for analysis (rs41176, rs229527, rs313841, rs941576, rs1048956, rs1456988, rs2194225, rs4422634, rs6043409, rs6427859, rs7202877, rs7576541, rs9585056, rs10116772, rs11980379).

\section{Results}

SLE- and pSS-associated eQTLs in B cells

We performed a cis eQTL analysis in B cells from a total of 287 healthy volunteers in $2 \mathrm{Mb}$ genomic regions centered on 22 non-HLA SNPs with reported genome-wide association with SLE or pSS (Table 1). Ten SNPs displayed significant eQTL effects (FDR <0.05), affecting a total of 16 different genes (Table 2). rs13277113 and rs922483 are in high LD $\left(r^{2}=0.83\right)$ and both displayed eQTL effects of similar magnitude for FAM167A and BLK. For FAM167A, SMG7, IRF5, SNRPC, TMEM80, and FDFT1, different Illumina probes mapping to the same gene transcripts generated similar results. Our results agree with the direction of the eQTL effect in relation to the risk alleles reported in other studies [17, 24, 36, 37], with the exception of IRF5 in relation to the rs4728142 risk allele as reported in the GTEx database.

We found three eQTL associations which, to our knowledge, have not been published before for any cell type nor has any SNP with $r^{2}>0.8$ been reported for the same eQTL effect: rs7574865-INPP1, rs7574865$M Y O 1 B$, and rs4938573-CD3D. rs11755393-SNRPC and rs4963128-PHRF1 were only reported in the GTEx database [36], however, not in any immune related tissue and are thus novel observations for the immune compartment and B cells (Table 2, Additional file 4: Figure S2). For rs4938573, an eQTL effect on two genes in the same region, TREH and AP002954, has been reported in liver tissue [36] and tibial nerve as well as thyroid gland, respectively [36].

In summary, we identified five novel eQTL associated genes in B cells for SLE- and pSS-related genetic polymorphisms and confirmed 11 previously reported eQTL associations.

\section{Differential SLE- and pSS-associated eQTL effects in males and females}

With the aim of testing whether there are eQTL effects of SLE- and pSS-associated SNPs that differ significantly between males and females (i.e., either an eQTL effect that is stronger or exists only in one of the sexes, or eQTL effects in both sexes, but with differing directions), we applied a linear model including the SNP genotype and the sex of the subject (125 males and 162 females in our study) as separate terms, as well as an interaction term for the joint effect of SNP genotype and sex. By analyzing the significance of the "SNP genotype * Sex" interaction coefficient, we identified seven SLEand/or pSS-associated SNPs with eQTL effects on six different genes which differed significantly between 
Table 2 SLE- and/or pSS-associated eQTLs in B cells

\begin{tabular}{|c|c|c|c|c|c|c|c|}
\hline Chr & SNP & $\begin{array}{l}\text { Reported gene(s) } \\
\text { associated with SLE/pSS }\end{array}$ & eQTL gene ${ }^{a}$ & $\begin{array}{l}\text { Expression } \\
\text { vs risk allele }\end{array}$ & $P$ & FDR & Reference \\
\hline 8 & rs13277113 & BLK/FAM167A & FAM167A & Increased & $2.13 \times 10^{-71}$ & $4.12 \times 10^{-69}$ & $\begin{array}{l}\text { Hom et al. [12], Lessard et al. [17], } \\
\text { Fairfax et al. [24], GTEx v6 [36] (immune) }\end{array}$ \\
\hline 8 & rs922483 & BLKVFAM167A & FAM167A & Increased & $5.17 \times 10^{-71}$ & $5.00 \times 10^{-69}$ & $\begin{array}{l}\text { Hom et al. [12], Lessard et al. [17], } \\
\text { Fairfax et al. [24], GTEx v6 [36] (immune) }\end{array}$ \\
\hline 7 & rs4728142 & IRF5/TNPO3 & IRF5 & Decreased & $2.69 \times 10^{-20}$ & $1.04 \times 10^{-18}$ & Fairfax et al. [24], GTEx v6 [36] (immune) \\
\hline 1 & rs12753665 & NCF2 & SMG7 & Decreased & $2.69 \times 10^{-19}$ & $8.69 \times 10^{-18}$ & Fairfax et al. [24], GTEx v6 [36] (immune) \\
\hline 8 & rs13277113 & BLK/FAM167A & $B L K$ & Decreased & $7.19 \times 10^{-15}$ & $1.99 \times 10^{-13}$ & $\begin{array}{l}\text { Hom et al. [12], Lessard et al. [17], } \\
\text { Fairfax et al. [24], GTEx v6 [36] (immune) }\end{array}$ \\
\hline 8 & rs922483 & BLK/FAM167A & $B L K$ & Decreased & $1.04 \times 10^{-12}$ & $2.51 \times 10^{-11}$ & $\begin{array}{l}\text { Hom et al. [12], Lessard et al. [17], } \\
\text { Fairfax et al. [24], GTEx v6 [36] (immune) }\end{array}$ \\
\hline 11 & rs4963128 & IRF7/PHRF1/KIAA1542 & IRF7 & Increased & $1.97 \times 10^{-7}$ & $4.24 \times 10^{-6}$ & Westra et al. [37] \\
\hline 2 & rs7574865 & STAT4 & INPP1 & Increased & $6.05 \times 10^{-5}$ & $1.17 \times 10^{-3}$ & Not reported ${ }^{b}$ \\
\hline 6 & rs11755393 & UHRF1BP1 & SNRPC & Decreased & $8.33 \times 10^{-5}$ & $1.54 \times 10^{-3}$ & GTEx v6 [36] (not immune) \\
\hline 7 & rs4728142 & IRF5/TNPO3 & TNPO3 & Decreased & $2.18 \times 10^{-4}$ & $3.83 \times 10^{-3}$ & Westra et al. [37] \\
\hline 11 & rs4963128 & IRF7/PHRF1/KIAA1542 & $\begin{array}{l}\text { TMEM80 (probe picks } \\
\text { up } 4 \text { transcripts) }\end{array}$ & Decreased & $2.37 \times 10^{-4}$ & $3.99 \times 10^{-3}$ & $\begin{array}{l}\text { Lappalainen et al. [21], Westra et al. [37], } \\
\text { GTEx v6 [36] (immune) }\end{array}$ \\
\hline 2 & rs7574865 & STAT4 & MYO1B & Decreased & $8.83 \times 10^{-4}$ & 0.014 & Not reported ${ }^{b}$ \\
\hline 11 & rs4963128 & IRF7/PHRF1/KIAA1542 & $\begin{array}{l}\text { TMEM80 (probe picks } \\
\text { up } 2 \text { transcripts) }\end{array}$ & Decreased & $1.45 \times 10^{-3}$ & 0.023 & $\begin{array}{l}\text { Lappalainen et al. [21], Westra et al. [37], } \\
\text { GTEx v6 [36] (immune) }\end{array}$ \\
\hline 7 & rs10488631 & IRF5/TNPO3 & IRF5 & Decreased & $2.00 \times 10^{-3}$ & 0.028 & Lappalainen et al. [21] \\
\hline 11 & rs4938573 & CXCR5 & $C D 3 D$ & Increased & $2.12 \times 10^{-3}$ & 0.028 & Not reported ${ }^{b}$ \\
\hline 8 & rs922483 & BLK/FAM167A & $\begin{array}{l}\text { FDFT1 (probe picks } \\
\text { up } 14 \text { transcripts) }\end{array}$ & Increased & $2.12 \times 10^{-3}$ & 0.028 & Zeller et al. [61] \\
\hline 6 & rs11755393 & UHRF1BP1 & UHRF1BP1 & Increased & $2.26 \times 10^{-3}$ & 0.028 & GTEx v6 [36] (immune) \\
\hline 11 & rs4963128 & IRF7/PHRF1/KIAA1542 & PHRF1 & Decreased & $3.00 \times 10^{-3}$ & 0.036 & GTEx v6 [36] (not immune) \\
\hline 8 & rs13277113 & BLK/FAM167A & $\begin{array}{l}\text { FDFT1 (probe picks } \\
\text { up } 11 \text { transcripts) }\end{array}$ & Increased & $3.31 \times 10^{-3}$ & 0.038 & Zeller et al. [61] \\
\hline 11 & rs4963128 & UHRF1BP1 & $\angle R R C 56$ & Increased & $3.60 \times 10^{-3}$ & 0.040 & Westra et al. [37] \\
\hline 3 & rs6445975 & PXK & DNASE1L3 & Decreased & $4.53 \times 10^{-3}$ & 0.049 & Fairfax et al. [24] \\
\hline
\end{tabular}

Chr chromosome, SNP single nucleotide polymorphism, eQTL expression quantitative locus, $P p$ value, FDR false discovery rate

${ }^{\mathrm{a}}$ For significant eQTLs where different probes detected the same transcript, only the result for the most significant probe is included in the table

${ }^{b}$ For rs7574865 no other genes in the region have been reported; for rs4938573 a cis-eQTL effect was reported for TREH in liver and for AP002954.6 in the tibial nerve and the thyroid gland (GTeX, [36])

males and females (FDR <0.05) and with substantial effect sizes reflected by $\beta$ values up to 0.66 (range -1 to 1) (Table 3).

The joint effect of "SNP genotype "Sex" contributed more significantly to the linear model than the single effect of "SNP genotype" for the expression of all these six genes. For rs4637409-SLC39A8, rs13277113-CTSB, and rs4938573-ARCN1, the single effect of "Sex" to the linear model was comparable to the effect of "SNP genotype * Sex," while the contribution of the interaction term was markedly more significant for rs10036748-CD74, rs6445975-PXK, rs922483-CTSB, and rs12753665-DHX9 than any of the single coefficient contributions. Figure 1 shows the genomic maps for the significantly differing eQTL effects in females compared to males. rs4637409 is located in an intron of BANK1; however, the differential eQTL effect in males and females was observed for
$S L C 39 A 8$, the first gene located approximately $400 \mathrm{~kb} \mathrm{3'}$ of BANK1 (Fig. 1a). CD74 is located almost $1 \mathrm{Mb}$ away from rs 10036748 (Fig. 1b). PXK is differentially regulated in a sex-dependent manner by rs6445975, located in an intron of the same gene (Fig. 1c), for which a sexindependent eQTL effect of rs6445975 has been published previously $[21,36]$. CTSB, ARCN1, and DHX9 are located $250 \mathrm{~kb}-1 \mathrm{Mb}$ from the disease risk SNP (Fig. 1d-f). In males, the SLE risk alleles of rs4637409 and rs10036748 were associated with higher expression of SLC39A8 and CD74, respectively, while the direction of this eQTL effect was the opposite in females, where the SLE risk alleles were associated with lower gene expression (Fig. 2a, b). rs6445975 showed no eQTL effect in males, while in females the SLE risk allele was associated with higher expression of PXK (Fig. 2c). The pSS risk alleles of rs922483 and rs13277113 indicate an effect 
Table 3 SLE- and/or pSS-associated SNPs displaying differential eQTL effects in females compared to males

\begin{tabular}{|c|c|c|c|c|c|c|c|c|c|c|}
\hline $\mathrm{Chr}$ & SNP & $\begin{array}{l}\text { Reported gene(s) } \\
\text { associated with SLE/pSS }\end{array}$ & eQTL Gene & Probe ID & Intercept & P SNP & P Sex & P SNP $\times$ Sex & $\beta$ value SNP $\times$ Sex & FDR SNP $\times$ Sex \\
\hline 4 & rs4637409 & BANK1 & SLC39A8 & ILMN_1695316 & $8.13 \times 10^{-3}$ & 0.016 & $1.20 \times 10^{-4}$ & $2.10 \times 10^{-4}$ & 0.66 & $7.90 \times 10^{-3}$ \\
\hline 5 & rs10036748 & TNIP1 & CD74 & ILMN_2379644 & 0 & 0.025 & 0.638 & $2.40 \times 10^{-3}$ & -0.55 & 0.019 \\
\hline 3 & rs6445975 & PXK & PXK & ILMN_1815063 & $1.34 \times 10^{261}$ & 0.261 & 0.020 & $6.00 \times 10^{-3}$ & -0.54 & 0.022 \\
\hline 8 & rs922483 & BLKJFAM167A & $C T S B$ & ILMN_1696360 & $2.85 \times 10^{-305}$ & 0.104 & 0.257 & $6.90 \times 10^{-3}$ & 0.51 & 0.028 \\
\hline 8 & rs13277113 & BLKJFAM167A & $C T S B$ & ILMN_1696360 & $1.26 \times 10^{-232}$ & 0.068 & $5.00 \times 10^{-3}$ & $8.60 \times 10^{-3}$ & 0.50 & 0.029 \\
\hline 11 & rs4938573 & CXCR5 & ARCN1 & ILMN_1699703 & 0.065 & 0.099 & 0.014 & 0.029 & 0.46 & 0.042 \\
\hline 1 & rs12753665 & NCF2 & $D H \times 9$ & ILMN_1690965 & 0.442 & 0.126 & 0.158 & 0.013 & -0.45 & 0.044 \\
\hline
\end{tabular}

Chr chromosome, SNP single nucleotide polymorphism, $e Q T L$ expression quantitative locus, $P p$ value, $\beta$ value regression coefficient, FDR false discovery rate

of higher expression in males while no effect was observed in females (Additional file 5: Figure S3a, b). For $A R C N 1$, the pSS risk allele of rs4938573 was associated with higher expression in females, but not in males (Additional file 5: Figure S3c). Higher expression of DHX9 was associated with the SLE risk allele of rs12753665 in females, while the effect was opposite in males (Additional file 5: Figure S3d).

In order to corroborate the findings, we performed the interaction analysis for the sex eQTLs and their corresponding genes, but substituting the SNP with one in high $\left(r^{2}>0.8\right)$ or moderate $\left(r^{2}>0.5\right) \mathrm{LD}$, if there were none available with high LD. Six proxy SNPs meeting the criteria were available in the data set. With the exception of rs2077579-ARCN1 (proxy for rs12753665, $p=0.06$ ), all the other SNPs show a significant SNP*sex interaction term and thus confirm the sex-eQTL results (Additional file 3: Table S2).

To investigate if the identified sex-influenced eQTLs are specific to B cells, we analyzed whether the SNPs with significant SNP*sex interaction terms acted as sex eQTLs in other cells or in tissue. For the analysis, we utilized a dataset generated from purified CD14 ${ }^{+}$monocytes of 432 genotyped individuals [25] in a similar manner for the analysis in the B cell dataset, as well as the online sex eQTL analysis tool (http:// 52.37.206.146:8000/) for whole blood based on data from Kukurba et al. [27]. No significant sex-biased regulation of eQTLs by the analyzed SNPs was found (Additional file 6: Table S3), demonstrating cell specificity of the sex-influenced eQTL effects.

The findings of sex-biased eQTLs among susceptibility SNPs for a disease could be a general phenomenon and not connected with a biased sex prevalence of the disease. As a comparison, we therefore analyzed whether there are sex-influenced eQTLs for SNPs associated with autoimmune disease with lesser difference in frequency of affected females/males. For this purpose, we chose type 1 diabetes (T1D), for which the female:male ratio is approximately $1: 1$ in children [38], and the incidence in adults is 1.5 times higher in men than women ( $>15$ years of age) [39]. We analyzed the 15 SNPs with a reported genome-wide association with T1D but not concomitantly associated with other diseases with greater differences in female:male ratios [35]. Of these 15, no SNP was associated with a sex-influenced eQTL at the same order of magnitude as the top ones in pSS/SLE, and more importantly, the effect size was lesser for all than for the pSS/SLE-associated SNPs (Additional file 7: Table S4).

In conclusion, we here identify six genes the expression of which is differentially regulated in females compared to males depending on genotype of SLE/pSSassociated SNPs, demonstrating the occurrence of sexspecific eQTL effects of SLE/pSS-associated genetic polymorphisms in defined cells and pinpointing possible genes and pathways which could begin explaining the sex difference in these diseases. We also show that in disease with lower sex bias in prevalence such as T1D, sex eQTLs are less likely to be found among susceptibility SNPs and mediate less effect.

\section{Discussion}

In the present study, we used a directed approach to address the question whether genetic polymorphisms associated with SLE and pSS may contribute to the difference in risk for disease between women and men. We performed an eQTL analysis of SLE and pSS risk loci in primary $\mathrm{CD}_{19}{ }^{+} \mathrm{B}$ cells, and subsequently narrowed down the question to studying how the context of sex influences eQTL effects of the disease-associated SNPs in $B$ cells. We identified five novel sex-independent eQTLs in B cells with strong effects, which to our knowledge have not been reported previously. In addition, we found sex-influenced eQTL effects for seven of the SLE/ pSS SNPs with differential allelic expression effects of six genes between women and men. Four of the sexdependent effects were exerted by SNPs that also had sex-independent effects on other genes in the same region. By using a cell type specific dataset, we are limiting the spectrum of possible regulatory effects of the disease-associated SNPs and can obtain a more specific 


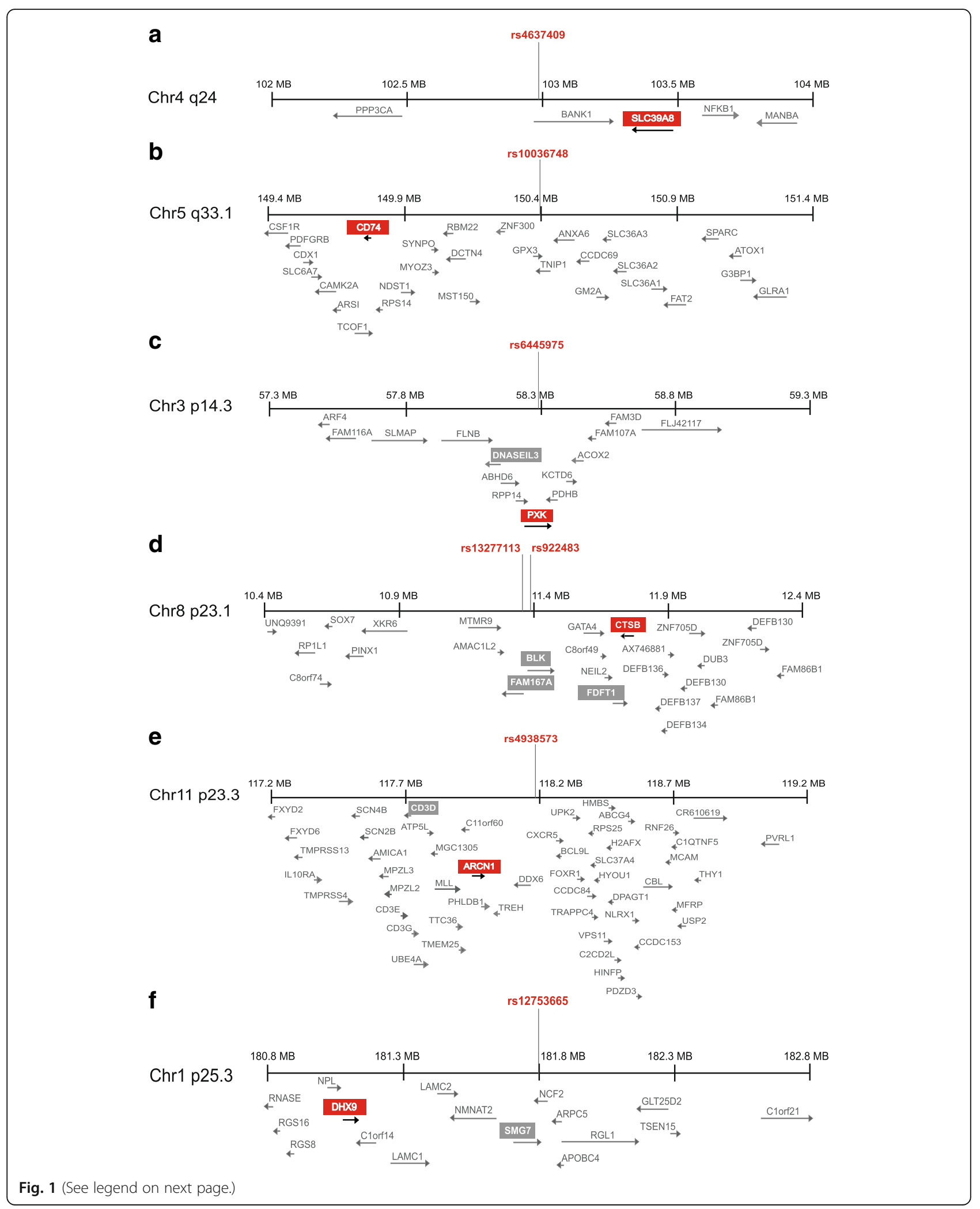


(See figure on previous page.)

Fig. 1 Chromosome and DNA strand location of SNPs with significant sex-influenced eQTL effect and surrounding genes in a $\pm 1 \mathrm{Mb}$ interval. Genes with significantly different sex-influenced expression are labeled in red, and genes with non-sex influenced expression are labeled gray. a rs4637409, proxy SNP $\left(r^{2}=1\right.$ ) for the SLE-associated rs10516487 (BANK1 locus) with a sex-dependent eQTL effect on SLC39A8 expression, located on chromosome 4, b rs10036748, proxy SNP $\left(r^{2}=1\right)$ for the SLE-associated rs7708392 (TNIP1 locus) with a sex-dependent eQTL effect on CD74 expression, located on chromosome 5. c The SLE-associated rs6445975 (PXK locus), with a sex-dependent eQTL effect on PXK expression, located on chromosome 3. d rs922483 (proxy SNP for the pSS-associated rs2736345, $r^{2}=1$ ) and the SLE-associated rs13277113 (BLK/FAM167A locus) with sex-dependent eQTL effects on CTSB expression. e rs4938573, proxy SNP $\left(r^{2}=0.865\right)$ for the pSS-associated rs7119038 (CXCR5 locus) with a sex-dependent eQTL effect on ARCN1 expression. f rs12753665, proxy SNP $\left(r^{2}=0.821\right)$ for the SLE-associated rs10911363 (NCF2 locus) with a sex-dependent eQTL effect on DHX9 expression

signal. Thus, we have been able to identify novel eQTLs in B cells, not reported previously, likely due to lacking power, mixed or other cell types analyzed in preceding studies. The specificity of the observation was also confirmed in that the signals were not present in monocytes or whole blood. By taking only taking the top SLE/pSS- associated SNPs into consideration for the cis-eQTL analysis, we were able to minimize the number of tests, and thus the number of type II errors that may arise from multiple comparisons. Interestingly, sex-influenced eQTL effects were substantially less significant and with less effect size for polymorphisms associated with type 1
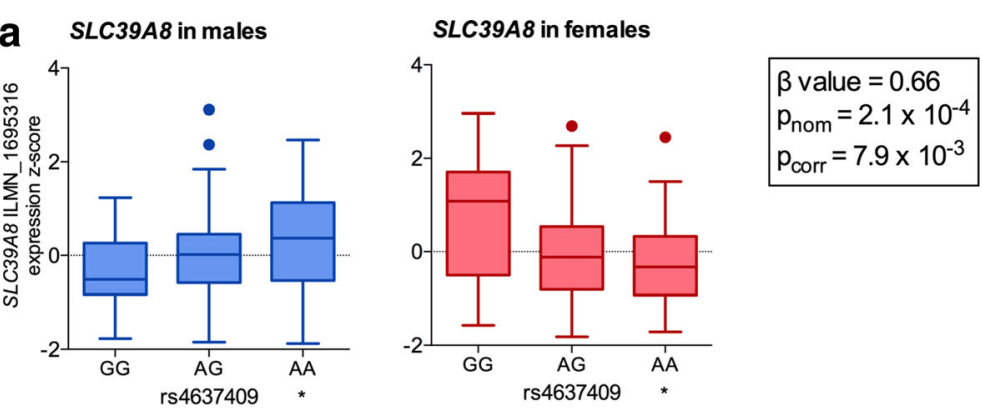

\section{b}

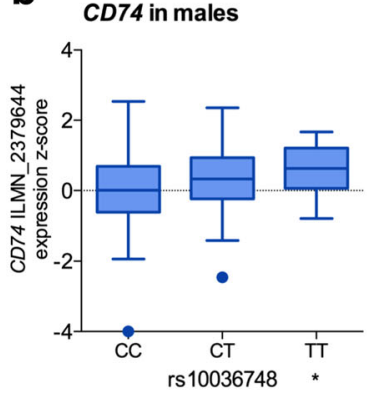

CD74 in females
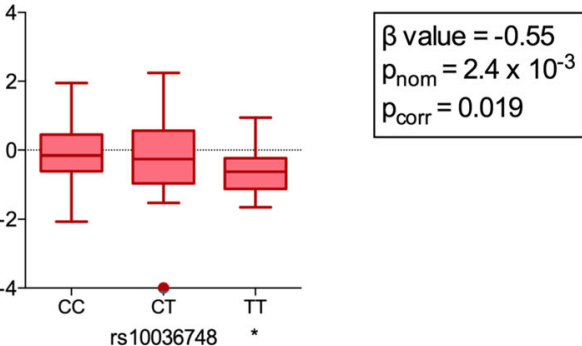

C
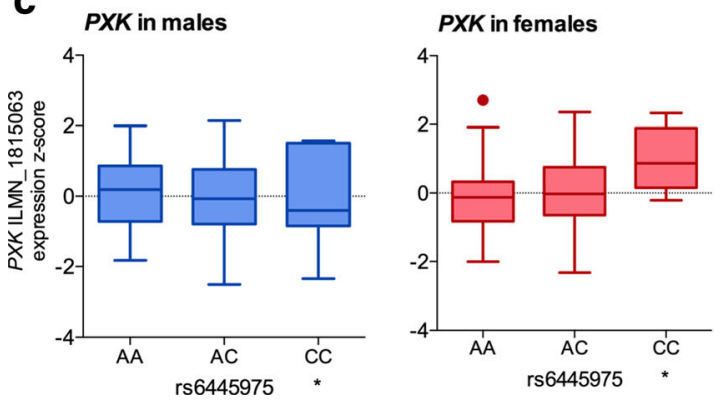

Fig. 2 eQTL effects of disease polymorphisms differ between males (left column, blue boxes) and females (right column, red boxes). a Genotypic effects of the proxy SNP rs4637409 $\left(r^{2}=1\right)$ for the SLE-associated rs 10516487 (BANK1 locus) on expression of SLC39A8 (ILMN_1695316 probe) in males $(p=0.041)$ and females $(p=0.052)$. $\mathbf{b}$ Genotypic effects of the proxy SNP rs $10036748\left(r^{2}=1\right)$ for the SLE-associated rs7708392 (TNIP1 locus) on expression of CD74 (ILMN_2379644 probe) in males $(p=0.065)$ and females $(p=0.048)$. c Genotypic effects of the SLE-associated rs6445975 (PXK locus) on expression of PXK (ILMN_1815063 probe) in males $(p=0.60)$ and females $(p=0.0029)$. The rightmost genotype group in each graph denotes the homozygous group of the disease risk allele and is marked by an asterisk. Statistics in boxes represent the SNP * Sex interaction term analysis 
diabetes, indicating that a high amount of sex-specific eQTL effects of disease-associated polymorphisms is specific for diseases with large differences in the frequency of affected females/males.

Interestingly, the sex-interacting eQTLs detected in this study displayed mostly opposing directions of their effects on expression in men compared to women. While similar observations have been reported previously [28], the underlying mechanisms remain unknown. When considering the pSS/SLE SNPs that showed a significant sex-specific eQTL, most of them are located in non-coding or intergenic regions. These polymorphisms could be responsible for repression or enhancement of gene expression. Sex hormones, through their intracellular receptors, can act both as transcription factors and chromatin modifiers, and the bi-directional sex eQTL effect could be brought about both by direct genetic differences in the receptor binding sites, enhancer elements or their surrounding sequences. One possible scenario is that the gene is regulated by both male and female hormones, and that their binding is affected by the disease-associated SNP. In such a scenario, the diseaseassociated allele could simply increase binding of e.g. female-specific factors involved in transcription, but decrease the binding of male-specific factors, while the alternative allele has the opposite effect. It is also possible that the disease-associated polymorphisms modulate the recruitment of the regulatory machinery via more distant epigenetic mechanisms, leading to differences in the expression of nearby genes through a pathway that is differentially regulated between the sexes, most probably indirectly orchestrated by sex hormones. Notably, studies have indicated that approximately two thirds of genes differentially expressed between sexes lack androgen or estrogen response elements and were not under direct influence of sex hormones, confirming the complexity of sexually dimorphic gene expression [40].

Whether the genes affected by the identified eQTLs are causal in SLE and/or pSS pathogenesis remains to be investigated. Out of the previously unpublished genes regulated by sex-independent mechanisms, INPP1, Inositol polyphosphate-1-phosphatase, is one of the enzymes involved in phosphatidylinositol signaling pathways [41], while $M Y O 1 B$, Myosin Ib, is reported to be involved in morphology and protein transport within multi-vesicular sorting endosomes [42]. Of potential interest is the eQTL affecting expression of SNRPC. This eQTL has not been studied before in the context of SLE. The small nuclear ribonucleoprotein polypeptide $\mathrm{C}$ $(S N R P C)$ gene encodes one of the specific protein components of the U1 small nuclear ribonucleoprotein (snRNP) required for formation of the spliceosome. Autoantibodies to these components are frequently found in autoimmune connective tissue disorders, mainly in SLE [43, 44]. Association signals in the SNRPC region have been shown to contribute to the association signal from the $H L A$ region in SLE [45].

The most significant eQTL effect difference between females and males was found for rs4637409 (proxy SNP for rs10516487 in the BANK1 locus) and the expression of SLC39A8. In females, presence of the SLE risk allele A was correlated with lower expression of SLC39A8, while the effect was opposite in males. The SLC39A8 (Solute carrier family 39 member 8 ) gene encodes a manganese and zinc transmembrane transporter localized mainly at the cell membrane, but also at the lysosomal and mitochondrial membranes [46]. SLC39A8 expression is under transcriptional control of the $N F K B$ pathway. While its role in B cells has not been defined, it has been shown to be induced in other immune cells upon microbial challenge, leading to increased intracellular zinc levels [47]. In lung epithelium, SLC39A8 was found to be essential for zinc-mediated protection against stress-induced cytotoxicity at the onset of inflammation [48]. Reduced expression of SLC39A8 in female carriers of the SLE-associated rs10516487 risk allele could potentially lead to enhanced inflammatory stress-induced cell damage and increased exposure of intracellular self-antigens.

The CD74 gene encodes the class II invariant chain, and is well known for its function as a chaperone, which prevents binding of peptides to the MHC class II molecules in the endoplasmic reticulum (ER), promotes their exit from the ER, directing it into the endocytic compartments, and contributes to peptide editing prior to antigen presentation [49]. CD74 has also been shown to be required for $\mathrm{B}$ cell maturation and function [50], and plays an additional role as an accessory signaling molecule on the surface of antigen-presenting cells [51]. In the present study, we found decreased CD74 expression in B cells from females carrying the risk allele of rs10036748 (proxy SNP for the SLE- and pSS-associated rs7708392 in the TNIP1 locus), which functionally may relate to an altered regulation of the peptide repertoire presented by MHC II. In males, the same allele was associated with increased $C D 74$ expression.

Regarding functional consequences of the $P X K$ region SNP, one study has reported that it influenced the rate of BCR internalization, and that subjects carrying the risk haplotype had a decreased rate of BCR internalization, a process known to impact B cell survival and cell fate [52]. CTSB encodes Cathepsin B, a lysosomal cysteine protease with a poorly studied function, but which potentially may play a role in protein turnover [53]. The protein encoded by $A R C N 1$ encodes a subunit of the COPI intracellular trafficking system and mutations in this gene cause a developmental craniofacial syndrome [54]. No associations with SLE or any other autoimmune 
diseases have been reported for CTSB and ARCN1. The RNA helicase A, encoded by the $D H X 9$ gene, is a known autoantibody-target in SLE [55]. Interestingly, this gene was first discovered in Drosophila as a gene which when mutated caused lethality in male zygotes and was found to play a role in X-chromosome dosage compensation in males [56, 57].

A limitation of the study is the small sample size, although to the best of our knowledge there are no other larger or similarly sized datasets currently available in terms of simultaneous genome-wide genotyping and gene expression data from purified B cells. Further limitations are the lack of information regarding age of the study subjects or other possibly relevant covariates to take into account. However, PCA analysis of ancestry informative markers did not identify any significant vectors, indicating the results are not due to hidden population substructures.

We observed SNPs with both sex-independent eQTL effects for one or several genes, while displaying a sexbiased eQTL effect on another gene. In multifactorial, multigenic diseases such as SLE, a wide range of factors are expected to influence the process leading to overt clinical disease. Genes affected by regulatory SNPs in different contexts, such as different tissue, inflammation and hormonal stimulus are likely influencing the pathogenesis either as a more or less simultaneous cascade at some point, or as single events spanning over a period of time. Theoretically, all those events could be necessary for the eventual development of disease. Thus, it is not surprising to find that one polymorphism or a haplotype could regulate different genes in different contexts, however of equal relevance in the pathogenesis.

\section{Conclusion}

In contrast to previous studies of how sex impacts the pathogenetic mechanisms in SLE, pSS, or other autoimmune diseases, we investigated the aspect of interaction between genetic risk loci and sex as a risk factor in leveraging expression of genes in cis in B cells. This approach identified several unknown sex-influenced eQTL effects of the disease-associated genetic polymorphisms, and provides an initial insight into how gene-sex interactions may contribute to the sex-bias in systemic autoimmune diseases. Our main finding is that gene expression will be different if the polymorphism is in the context of a female or male cells, and this may explain why the risk for disease is much higher in females, although the frequency of carriers does not differ between women and men. Our findings will also help designing mechanistic studies intended to pinpoint causal pathways in SLE and pSS pathogenesis to gain functional understanding of why the disease risk differs between men and women.

\section{Additional files}

\begin{abstract}
Additional file 1: Figure S1. "Principal component analysis". Genotypes for 3736 ancestry informative SNPs were used for a principal component analysis (PCA) by running the smartpca program from Eigensoft using standard settings. Five population outliers were identified. Due to this low number, none of them was removed from the analysis. None of the PCs was significant ( $p=0.68,0.75,0.94,0.95,0.95$ for the first 5 PCs). (a) scatter plots of first four PCs. (b) scatterplots of first two PCs stratified by genotype and sex for each of six markers with significant sex eQTL effects. The plot for a seventh SNP, rs922483, is not shown as the result was highly similar to $r 513277113\left(r^{2}=0.83\right)$. (PDF $310 \mathrm{~kb}$ )
\end{abstract}

Additional file 2: Table S1. "Number of probes analyzed per SLE/pSS SNP". List of SLE/pSS SNPs included in the eQTL analysis, with corresponding numbers of gene expression probes analyzed per SNP. Sheet 2 lists the individual probes and corresponding genes. (XLSX $18 \mathrm{~kb}$ )

Additional file 3: Table S2. "Analysis of sex-specific eQTLs using LD SNPs". SNP*sex Interaction analysis of SNPs in high $\left(r^{2}>0.8\right)$ or moderate $\left(r^{2}>0.5\right)$ LD with the sex-interacting SLE eQTLs. (XLSX $\left.10 \mathrm{~kb}\right)$

Additional file 4: .Figure S2 "eQTL effects of SLE and/or pSS-associated polymorphisms in B cells". eQTL effects of SLE and/or pSS-associated SNPs in primary naïve B cells. The $p$-value represents the significance in differential expression between the homozygous group of the non-risk allele and the homozygous group of the risk allele. The rightmost genotype group in each graph denotes the homozygous group of the disease risk allele and is marked by *. (PDF $121 \mathrm{~kb}$ )

Additional file 5: Figure S3. "Sex-specific eQTL effects of SLE and/or pSS associated polymorphisms in B cells". eQTL effects of disease polymorphisms differ between males (left column, blue boxes) and females (right column, red boxes). (a) Genotypic effects of the proxy SNP rs922483 ( $\left.r^{2}=1\right)$ for the pSS-associated rs2736345 (BLK/C8orf13 locus) on expression of CTSB (ILMN_1696360 probe) in males ( $p=0.059)$ and females $(p=0.12$ ). (b) Genotypic effects of the SLE-associated rs13277113 (BLKVC8orf13 locus) on expression of CTSB (ILMN_1696360 probe) in males $(p=0.16)$ and females $(p=0.19)$. (c) Genotypic effects of the proxy SNP rs4938573 $\left(r^{2}=0.865\right)$ for the pSS-associated rs7119038 (CXCR5 locus) on expression of ARCN1 (ILMN_1699703 probe) in males ( $p=0.28)$ and females $(p=0.15)$. (d) Genotypic effects of the proxy SNP rs 12753665 $\left(r^{2}=0.821\right)$ for the SLE-associated rs10911363 (NCF2 locus) on expression of DHX9 (ILMN 1690965 probe) in males $(p=0.15)$ and females $(p=0.06)$. The rightmost genotype group in each graph denotes the homozygous group of the disease risk allele and is marked by *. Statistics in boxes represent the Sex * SNP interaction term analysis. (PDF 391 kb)

Additional file 6: Table S3. "Sex-influenced eQTL effects in monocytes and whole blood". SNPS with SNP * sex interaction terms of FDR $<0.05$ analyzed for sex-influenced eQTL effects also in monocytes and whole blood. (DOCX $81 \mathrm{~kb}$ )

Additional file 7: Table S4. "Sex-interaction analysis of T1D susceptibility SNPs". List of SNP* sex interactions (only eQTLs with nominal $p<0.05$ are shown). Result from a linear regression cis-eQTL analysis with a sex-interaction term, including 15 SNPs associated with T1D and a total of 161 genes included from $1 \mathrm{Mb}$ regions surrounding the SNPs. (DOCX $68 \mathrm{~kb}$ )

\section{Acknowledgements}

We thank professor Julian C Knight and his research group at the University of Oxford for sharing genotype and expression data from primary B cells.

\section{Funding}

The study was supported by grants from the Swedish Research Council, the Heart-Lung Foundation, the Stockholm County Council, Karolinska Institutet, the Ragnar and Torsten Söderberg Foundation, the Swedish Rheumatism association, and the King Gustaf the V:th 80-year foundation.

\section{Availability of data and materials}

The data that support the findings of this study are available from Fairfax et al. [24] upon request. 


\section{Authors' contributions}

All authors met the criteria for authorship. ML and MWH designed the study; data was analyzed and interpreted by ML, JRS, TJ, GET, SB, DGC, TO, IK, and $\mathrm{MWH}$. ML, JIRS, and MWH wrote the manuscript, and all authors critically reviewed the article. All authors read and approved the final manuscript.

\section{Ethics approval and consent to participate}

The expression data was obtained from Fairfax et al. [24]. The Fairfax et al.'s study was approved by the Oxfordshire Research Ethics Committee (COREC reference 06/Q1605/55). All 288 volunteers gave their written consent.

\section{Consent for publication}

Not applicable

\section{Competing interests}

The authors declare that they have no competing interests.

\section{Publisher's Note}

Springer Nature remains neutral with regard to jurisdictional claims in published maps and institutional affiliations.

\section{Author details}

'Unit of Experimental Rheumatology, Department of Medicine, Karolinska University Hospital, Karolinska Institutet, Stockholm, Sweden. ${ }^{2}$ Unit of Neuroimmunology, Department of Clinical Neuroscience, Karolinska University Hospital, Karolinska Institutet, Stockholm, Sweden. ${ }^{3}$ Unit of Computational Medicine, Karolinska University Hospital, Karolinska Institutet, Stockholm, Sweden. ${ }^{4}$ Unit of Clinical Epidemiology, Department of Medicine, Karolinska University Hospital, Karolinska Institutet, Stockholm, Sweden. ${ }^{5}$ Science for Life Laboratory, 17121 Solna, Sweden. ${ }^{6}$ Mucosal and Salivary Biology Division, King's College London Dental Institute, London SE1 9RT, UK.

Received: 3 April 2017 Accepted: 9 October 2017

Published online: 25 October 2017

\section{References}

1. Mahdi H, Fisher BA, Kallberg H, et al. Specific interaction between genotype, smoking and autoimmunity to citrullinated alpha-enolase in the etiology of rheumatoid arthritis. Nat Genet. 2009:41:1319-24.

2. Too CL, Muhamad NA, llar A, et al. Occupational exposure to textile dust increases the risk of rheumatoid arthritis: results from a Malaysian population-based case-control study. Ann Rheum Dis. 2016;75:997-1002.

3. Sundqvist $E$, Sundstrom $P$, Linden $M$, et al. Epstein-Barr virus and multiple sclerosis: interaction with HLA. Genes Immun. 2012:13:14-20.

4. Hedstrom AK, Lima Bomfim I, Barcellos L, et al. Interaction between adolescent obesity and HLA risk genes in the etiology of multiple sclerosis. Neurology. 2014;82:865-72.

5. Whitacre CC. Sex differences in autoimmune disease. Nat Immunol. 2001;2 $777-80$

6. Helmick CG, Felson DT, Lawrence RC, et al. Estimates of the prevalence of arthritis and other rheumatic conditions in the United States. Part I. Arthritis Rheum. 2008;58:15-25.

7. Patel R, Shahane A. The epidemiology of Sjogren's syndrome. Clin Epidemiol. 2014;6:247-55.

8. Kvarnstrom M, Ottosson V, Nordmark B, Wahren-Herlenius M. Incident cases of primary Sjogren's syndrome during a 5-year period in Stockholm County: a descriptive study of the patients and their characteristics. Scand J Rheumatol. 2015;44:135-42

9. Hughes GC, Choubey D. Modulation of autoimmune rheumatic diseases by oestrogen and progesterone. Nat Rev Rheumatol. 2014;10:740-51.

10. Rubtsova K, Marrack P, Rubtsov AV. Sexual dimorphism in autoimmunity. J Clin Invest. 2015;125:2187-93

11. Selmi C, Brunetta E, Raimondo MG, Meroni PL. The X chromosome and the sex ratio of autoimmunity. Autoimmun Rev. 2012;11:A531-7.

12. Hom G, Graham RR, Modrek B, et al. Association of systemic lupus erythematosus with C8orf13-BLK and ITGAM-ITGAX. N Engl J Med. 2008:358:900-9.

13. International Consortium for Systemic Lupus Erythematosus G, Harley JB, Alarcon-Riquelme ME, et al. Genome-wide association scan in women with systemic lupus erythematosus identifies susceptibility variants in ITGAM, PXK, KIAA1542 and other loci. Nat Genet. 2008;40:204-10.
14. Gateva V, Sandling JK, Hom G, et al. A large-scale replication study identifies TNIP1, PRDM1, JAZF1, UHRF1BP1 and IL10 as risk loci for systemic lupus erythematosus. Nat Genet. 2009;41:1228-33.

15. Kozyrev SV, Abelson AK, Wojcik J, et al. Functional variants in the B-cell gene BANK1 are associated with systemic lupus erythematosus. Nat Genet. 2008:40:211-6.

16. Cunninghame Graham DS, Morris DL, Bhangale TR, et al. Association of NCF2, IKZF1, IRF8, IFIH1, and TYK2 with systemic lupus erythematosus. PLoS Genet. 2011;7:e1002341.

17. Lessard CJ, Li H, Adrianto I, et al. Variants at multiple loci implicated in both innate and adaptive immune responses are associated with Sjogren's syndrome. Nat Genet. 2013;45:1284-92.

18. Bentham J, Morris DL, Cunninghame Graham DS, et al. Genetic association analyses implicate aberrant regulation of innate and adaptive immunity genes in the pathogenesis of systemic lupus erythematosus. Nat Genet. 2015;47:1457-64.

19. Stranger BE, Forrest MS, Clark AG, et al. Genome-wide associations of gene expression variation in humans. PLoS Genet. 2005;1:e78.

20. Genomes Project C, Abecasis GR, Altshuler D, et al. A map of human genome variation from population-scale sequencing. Nature. 2010;467:1061-73.

21. Lappalainen $T$, Sammeth M, Friedlander MR, et al. Transcriptome and genome sequencing uncovers functional variation in humans. Nature. 2013;501:506-11.

22. Dixon AL, Liang L, Moffatt MF, et al. A genome-wide association study of global gene expression. Nat Genet. 2007;39:1202-7.

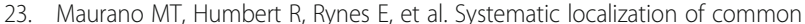
disease-associated variation in regulatory DNA. Science. 2012;337:1190-5.

24. Fairfax BP, Makino S, Radhakrishnan J, et al. Genetics of gene expression in primary immune cells identifies cell type-specific master regulators and roles of HLA alleles. Nat Genet. 2012;44:502-10.

25. Fairfax BP, Humburg $P$, Makino $S$, et al. Innate immune activity conditions the effect of regulatory variants upon monocyte gene expression. Science 2014;343:1246949.

26. Lee MN, Ye C, Villani AC, et al. Common genetic variants modulate pathogen-sensing responses in human dendritic cells. Science. 2014;343: 1246980.

27. Kukurba KR, Parsana P, Balliu B, et al. Impact of the $X$ chromosome and sex on regulatory variation. Genome Res. 2016;26:768-77.

28. Yao $C$, Joehanes $R$, Johnson $A D$, et al. Sex- and age-interacting eQTLs in human complex diseases. Hum Mol Genet. 2014;23:1947-56.

29. Johnson AD, Handsaker RE, Pulit SL, et al. SNAP: a web-based tool for identification and annotation of proxy SNPs using HapMap. Bioinformatics. 2008;24:2938-9.

30. Ward LD, Kellis M. HaploReg: a resource for exploring chromatin states, conservation, and regulatory motif alterations within sets of genetically linked variants. Nucleic Acids Res. 2012:40:D930-4.

31. Purcell $S$, Neale B, Todd-Brown K, et al. PLINK: a tool set for whole-genome association and population-based linkage analyses. Am J Hum Genet. 2007: 81:559-75.

32. Shabalin AA. Matrix eQTL: ultra fast eQTL analysis via large matrix operations. Bioinformatics. 2012;28:1353-8.

33. Li J, Tibshirani R. Finding consistent patterns: a nonparametric approach for identifying differential expression in RNA-Seq data. Stat Methods Med Res. 2013;22:519-36.

34. Storey JD, Tibshirani R. Statistical significance for genomewide studies. Proc Natl Acad Sci U S A. 2003:100:9440-5.

35. Onengut-Gumuscu S, Chen WM, Burren O, et al. Fine mapping of type 1 diabetes susceptibility loci and evidence for colocalization of causal variants with lymphoid gene enhancers. Nat Genet. 2015;47:381-6.

36. Consortium GT. The genotype-tissue expression (GTEx) project. Nat Genet. 2013:45:580-5

37. Westra HJ, Peters MJ, Esko T, et al. Systematic identification of trans eQTLs as putative drivers of known disease associations. Nat Genet. 2013:45:1238-43.

38. Soltesz G, Patterson CC, Dahlquist G, Group ES. Worldwide childhood type 1 diabetes incidence-what can we learn from epidemiology? Pediatr Diabetes. 2007;8(Suppl 6):6-14

39. Diaz-Valencia PA, Bougneres P, Valleron AJ. Global epidemiology of type 1 diabetes in young adults and adults: a systematic review. BMC Public Health. 2015:15:255.

40. Mayne BT, Bianco-Miotto T, Buckberry S, et al. Large scale gene expression meta-analysis reveals tissue-specific, sex-biased gene expression in humans. Front Genet. 2016;7:183. 
41. York JD, Veile RA, Donis-Keller H, Majerus PW. Cloning, heterologous expression, and chromosomal localization of human inositol polyphosphate 1-phosphatase. Proc Natl Acad Sci U S A. 1993;90:5833-7.

42. Salas-Cortes L, Ye F, Tenza D, et al. Myosin lb modulates the morphology and the protein transport within multi-vesicular sorting endosomes. J Cell Sci. 2005;118:4823-32.

43. Hassfeld W, Steiner G, Studnicka-Benke A, et al. Autoimmune response to the spliceosome. An immunologic link between rheumatoid arthritis, mixed connective tissue disease, and systemic lupus erythematosus. Arthritis Rheum. 1995;38:777-85.

44. Klein Gunnewiek JM, van de Putte LB, van Venrooij WJ. The U1 snRNP complex: an autoantigen in connective tissue diseases. An update. Clin Exp Rheumatol. 1997;15:549-60.

45. Armstrong DL, Zidovetzki R, Alarcon-Riquelme ME, et al. GWAS identifies novel SLE susceptibility genes and explains the association of the HLA region. Genes Immun. 2014;15:347-54.

46. Jenkitkasemwong S, Wang CY, Mackenzie B, Knutson MD. Physiologic implications of metal-ion transport by ZIP14 and ZIP8. Biometals. 2012;25:643-55.

47. Begum NA, Kobayashi M, Moriwaki Y, et al. Mycobacterium bovis BCG cell wall and lipopolysaccharide induce a novel gene, BIGM103, encoding a 7TM protein: identification of a new protein family having Zn-transporter and Zn-metalloprotease signatures. Genomics. 2002;80:630-45.

48. Besecker B, Bao S, Bohacova B, et al. The human zinc transporter SLC39A8 (Zip8) is critical in zinc-mediated cytoprotection in lung epithelia. Am J Physiol Lung Cell Mol Physiol. 2008;294:L1127-36.

49. Stumptner-Cuvelette $P$, Benaroch $P$. Multiple roles of the invariant chain in MHC class II function. Biochim Biophys Acta. 2002;1542:1-13.

50. Shachar I, Flavell RA. Requirement for invariant chain in B cell maturation and function. Science. 1996;274:106-8.

51. Naujokas MF, Morin M, Anderson MS, Peterson M, Miller J. The chondroitin sulfate form of invariant chain can enhance stimulation of $\mathrm{T}$ cell responses through interaction with CD44. Cell. 1993;74:257-68.

52. Vaughn SE, Foley C, Lu X, et al. Lupus risk variants in the PXK locus alter Bcell receptor internalization. Front Genet. 2014;5:450.

53. Sigloch FC, Knopf JD, Weisser J, et al. Proteomic analysis of silenced cathepsin B expression suggests non-proteolytic cathepsin B functionality. Biochim Biophys Acta. 1863;2016:2700-9.

54. Izumi K, Brett M, Nishi E, et al. ARCN1 mutations cause a recognizable craniofacial syndrome due to COPI-mediated transport defects. Am J Hum Genet. 2016;99:451-9.

55. Yamasaki Y, Narain S, Yoshida H, et al. Autoantibodies to RNA helicase A: a new serologic marker of early lupus. Arthritis Rheum. 2007:56:596-604.

56. Fukunaga A, Tanaka A, Oishi K. Maleless, a recessive autosomal mutant of Drosophila melanogaster that specifically kills male zygotes. Genetics. 1975; 81:135-41.

57. Kuroda MI, Kernan MJ, Kreber R, Ganetzky B, Baker BS. The maleless protein associates with the $\mathrm{X}$ chromosome to regulate dosage compensation in drosophila. Cell. 1991;66:935-47.

58. Graham RR, Cotsapas C, Davies L, et al. Genetic variants near TNFAIP3 on $6 q 23$ are associated with systemic lupus erythematosus. Nat Genet. 2008;40: 1059-61.

59. Lessard CJ, Adrianto I, Kelly JA, et al. Identification of a systemic lupus erythematosus susceptibility locus at 11p13 between PDHX and CD44 in a multiethnic study. Am J Hum Genet. 2011;88:83-91.

60. Lessard CJ, Adrianto I, Ice JA, et al. Identification of IRF8, TMEM39A, and IKZF3-ZPBP2 as susceptibility loci for systemic lupus erythematosus in a large-scale multiracial replication study. Am J Hum Genet. 2012;90:648-60.

61. Zeller T, Wild P, Szymczak S, et al. Genetics and beyond-the transcriptome of human monocytes and disease susceptibility. PLoS One. 2010;5:e10693.

\section{Submit your next manuscript to BioMed Central and we will help you at every step:}

- We accept pre-submission inquiries

- Our selector tool helps you to find the most relevant journal

- We provide round the clock customer support

- Convenient online submission

- Thorough peer review

- Inclusion in PubMed and all major indexing services

- Maximum visibility for your research

Submit your manuscript at www.biomedcentral.com/submit
Biomed Central 